\title{
Comparison between Hemiarthroplasty in the First Proximal Phalanx and Keller's Arthroplasty. A Finite Element Study.
}

\author{
Mario Alberto Madrid Pérez ${ }^{1}$, Javier Bayod López ${ }^{1}$ \\ ${ }^{1}$ Departamento de ingeniería mecánica. \\ Applied Mechanics and Bioengineering. Instituto de Investigación en Ingeniería de Aragón (I3A) \\ Universidad de Zaragoza, Mariano Esquillor s/n, 50018, Zaragoza, Spain. \\ Tel. +34-976762707, e-mail: $715181 @$ unizar.es
}

\begin{abstract}
Hallux valgus and hallux rigidus are the most common pathologies in the first ray of the foot. Arthroplasty can restore the mobility of the joint but is a destructive procedure. This paper presents three finite element analysis of the foot studying two different kinds of arthroplasty.
\end{abstract}

\section{Introduction}

Hallux valgus is the most common chronic pathology in the first ray of the foot. This is characterized by a deviation in varus of the first metatarsal to which is added a distal phalanx in valgus and in internal rotation. [1]. On the other hand, hallux rigidus is the second most common pathology in the first radius of the foot. This pathology is a degenerative arthritis of the first metatarsophalangeal joint which causes pain to the patient and is associated with limited movement, especially in dorsiflexion, and the appearance of osteophytes [2]. At present there is no technique that can correct all the pathological elements of the diseases described above, however the most common surgical procedures today are: osteotomy, chylectomy, arthrodesis and arthroplasty [3]. The presented work focuses on the subject of arthroplasty in the first metatarsophalangeal joint. In this paper, three models of finite elements of the foot are presented in the Toe-off stage. The first one analyzes a foot of a person of 36 years of age and 60 $\mathrm{kg}$ of weight free of any pathology. The second one analyzes the same foot after having undergone a hemiarthroplasty in the first proximal phalanx and the third of them analyzes the foot when a Keller arthroplasty has been developed

\section{Method}

For this Project the bones, cartilages, soft tissues, filling material and prosthesis were considered homogeneous, isotropic and linearly deformable bodies. The mechanical properties are defined in table 1. Regarding the border conditions, they are the same in the three models. A pre-tension force is added to the muscles as a result of the toe-off stage. Then a fixed suppor is used in; the insertion surface of the Achilles heel, the first and second proximal phalanx. In the distal phalanges of the lesser toes the rotation and translation in the vertical global axis of the model are restricted and then the load corresponding to the person's own weight in the toeoff position is added in the area where the tibial and fibula make contact with the talus. For more details of the boundary conditions of the model, the next reference can be consulted [4]. All models was created by the group of mechanical engeeniering of the Zaragoza university.The first model consists of 28 bones: 12 minor phalanges, 2 main phalanges, 5 metatarsals, 3 cuneiforms, scaphoids, cuboids, talus, calcaneus and two sesamoids embedded in the first metatarsal. A distinction was made between spongy bone and cortical bone, in order to provide different mechanical properties to each of them. The tetrahedral elements had an average size of $1 \mathrm{~mm}$ because with this element the problem of the convergence of the mesh is solved. This model contains some ligaments that were modeled as truss elements because they only suffer a displacement at their ends when the model is loaded, this model contains too some muscles and tendons that were modeled as beam elements because they suffer bending due to a pretension force as consecuense of to the toe-off stage. This model without implant contains 150594 nodes and 802294 elements.

The second model presented in this article analyzes the foot after having performed a hemiarthroplasty in the first proximal phalanx with the Anatoemics Phalangeal Prosthesis by Arthrex. To perform the model, the prosthesis was scanned with a 3D scanner provided by UNIZAR. A resection of 2 to 3 $\mathrm{mm}$ was performed in the proximal area of the first phalanx and then the implant was placed. The mesh of this second model was made with the software ICEM by ANSYS considering a maximum element size of $1 \mathrm{~mm}$ to solve the mesh convergence 
problem. Then MATLAB was used to add the soft tissues so that they are located in the nearest node of the new mesh with respect to the corresponding node of the old mesh. For this model some muscles and tendons were disinserted as a consequence of the arthroplasty. This model with implant contains 1836607 elements and 329159 nodes.

For this third model presented in this project, the same methodology was followed for the hemiarthroplasty, with the difference that Keller's arthroplasty does not use any prosthesis, that is, only the first proximal phalanx is resected and a filling material is added in said area. This model contains 412191 nodes and 2256585 elements.

\section{Results and conclutions}

For the first model presented in this article, the maximum and minimum major stresses appear in the talus in the area of Achilles heel insertion as well as in the first proximal phalanx. For the second and third radius of the foot the greatest concentration of principal absolute efforts occurs at the points of support. In the results of this model it is observed that the first distal phalanx acquires what is commonly known as the passive windlass mechanism (see figure 1). With respect to the second model, the stresses on the first radius of the foot decrease drastically so that the maximum absolute principal stresses appear at the points of support of the second and third proximal phalanges. For the implant studied, a maximum Von Mises's stress of $52 \mathrm{Mpa}$ is obtained. For the third model presented there is also a loss of load capacity for the first radius of the foot, leaving the absolute maximum stresses at the points of support of the second and third proximal phalanges, unlike with the model with hemiarthroplasty, Keller's arthroplasty presents greater displacements in the lesser toes and a lesser stress in the second ray of the foot. In the two models of arthroplasty developed in the present work a lost of the passive windlass mechanism is observed, because of the autor of this paper makes the recommendation to the physicians to make an assessment of the active windlass mechanism during the postoperative follow-up. The first ray of the foot gives the ability to adjust the march to terrains with irregular slopes. Therefore, this capacity may be diminished with the two kinds of hemiarthroplasty studied in this paper. Garcia-Aznar et al[5] have shown that the lesser toes overload is higher when the first ray is unloaded having thus a risk factor to induce metatarsalgia. On the othe hand Kurt F Konkel et al report that metatarsalgia can be an posible risk of the surgical procedure [6]. This study demonstrates that the two kinds of hemiarthroplasty generate an overload on the second ray of the foot.

\section{TABLES AND FIGURES.}

Table 1. Mechanic properties.

Mechanical properties of bones, soft tissues and prosthesis

\begin{tabular}{lcc}
\hline Tissues & Elasticity modulus $(\mathrm{Mpa})$ & Poisson's ratio $(\mathrm{v})$ \\
\hline Cortical bone & 17000 & 0.3 \\
Trabecular bone & 700 & 0.3 \\
Cartilage & 10 & 0.4 \\
Muscles & 450 & 0 \\
Thin ligaments & 260 & 0.3 \\
Plantar ligaments & 350 & 0.3 \\
Filling material & 1 & 0.45 \\
Prosthesis $(\mathrm{Co}-\mathrm{Cr})$ & 210000 & 0.29 \\
\hline
\end{tabular}

\section{REFERENCIAS}

[1] A. M. PEREA, L. MASON, and M. M. STEPHENS, "The pathogenesis of hallux valgus.," J. Bone Joint Surg. Am., vol. 93, no. 17, pp. 1650-61, 2011.

[2] J. ASUNCIÓN MÁRQUEZ and X. MARTÍN OLIVA, "Hallux rígidus: Etiología, diagnóstico, clasificación y tratamiento," Rev. Esp. Cir. Ortop. Traumatol., vol. 54, no. 5, pp. 321-328, 2010.

[3] S. FRANCISCO, "Cirugía percutánea del antepié."

[4] ENRIQUE MORALES ORCAJO, "Influencia de la geometría de la falange proximal en la formación de juanetes," 2012.

[5] J. M. GARCIA-AZNAR, "Load Transfer Mechanism for Different Metatarsal Geometries: A Finite Element Study," J. Biomech. Eng., vol. 131, no. 2, p. 021011, 2008.

[6] K. F. KONKEL, A. G. MENGER, and S. A. RETZLAFF, "Results of Metallic Hemi-Great Toe Implant for Grade III and Early Grade IV Hallux Rigidus," Foot Ankle Int., vol. 30, no. 7, pp. 653-660, 2009.
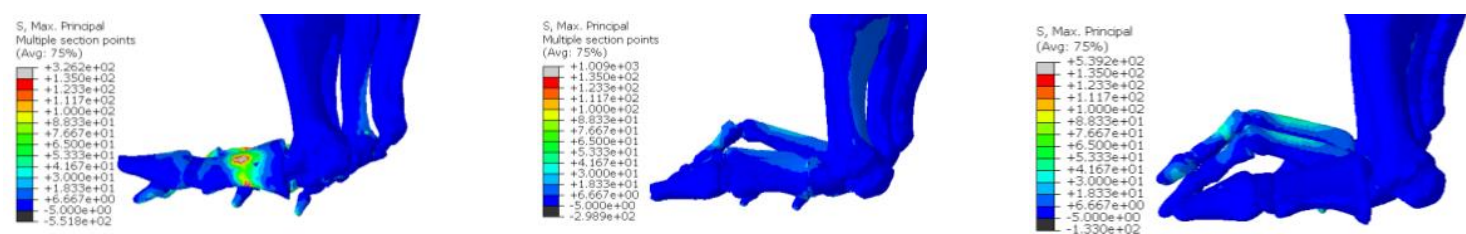

Figure 1. Lost of passive windlass mechanism (first distal phalanx) and maximum principal stresses in models. Left; healthy model. Midle; Hemiarhroplasty model. Right; Keller's arthroplasty model.

Revista "Jornada de Jóvenes Investigadores del I3A", vol. 6 (Actas de la VII Jornada de Jóvenes Investigadores del I3A - 8 de junio de 2018). ISSN 2341-4790. 